\title{
Correlation between Brain Reorganization, Ischemic Damage, and Neurologic Status after Transient Focal Cerebral Ischemia in Rats: A Functional Magnetic Resonance Imaging Study
}

\author{
Rick M. Dijkhuizen, ${ }^{1,2}$ Aneesh B. Singhal, ${ }^{2}$ Joseph B. Mandeville, ${ }^{1}$ Ona Wu, ${ }^{1}$ Elkan F. Halpern, ${ }^{3}$ Seth P. Finklestein, ${ }^{4}$ \\ Bruce R. Rosen, ${ }^{1}$ and Eng H. Lo ${ }^{2}$ \\ ${ }^{1}$ Athinoula A. Martinos Center for Biomedical Imaging, Department of Radiology, and ${ }^{2}$ Neuroprotection Research Laboratory, Departments of Radiology \\ and Neurology, Massachusetts General Hospital, Harvard Medical School, Charlestown, Massachusetts 02129, ${ }^{3}$ Data Analysis Group, Department of \\ Radiology, Massachusetts General Hospital, Harvard Medical School, Boston, Massachusetts 02114, and ${ }^{4}$ ViaCell Neuroscience Inc., Worcester, \\ Massachusetts 01605
}

The pattern and role of brain plasticity in stroke recovery has been incompletely characterized. Both ipsilesional and contralesional changes have been described, but it remains unclear how these relate to functional recovery. Our goal was to correlate brain activation patterns with tissue damage, hemodynamics, and neurologic status after temporary stroke, using functional magnetic resonance imaging (fMRI). Transverse relaxation time $\left(\mathrm{T}_{2}\right)$-weighted, diffusion-weighted, and perfusion MRI were performed at days $1(n=7), 3(n=7)$, and $14(n=7)$ after $2 \mathrm{hr}$ unilateral middle cerebral artery occlusion in rats. Functional activation and cerebrovascular reactivity maps were generated from contrast-enhanced fMRI during forelimb stimulation and hypercapnia, respectively. Before MRI, rats were examined neurologically. We detected loss of activation responses in the ipsilesional sensorimotor cortex, which was related to $\mathrm{T}_{2}$ lesion size ( $r=-0.858$ on day $3, r=-0.979$ on day $14 ; p<0.05$ ). Significant activation responses in the contralesional hemisphere were detected at days 1 and 3 . The degree of shift in balance of activation between the ipsilesional and contralesional hemispheres, characterized by the laterality index, was linked to the $\mathrm{T}_{2}$ and apparent diffusion coefficient in the ipsilesional contralesional forelimb region of the primary somatosensory cortex and primary motor cortex at day $1(r=-0.807$ and 0.782 , respectively; $p<0.05)$ and day $14(r=-0.898$ and -0.970 , respectively; $p<0.05$ ). There was no correlation between activation parameters and perfusion status or cerebrovascular reactivity. Finally, we found that the laterality index and neurologic status changed in parallel over time after stroke, so that when all time points were grouped together, neurologic status was inversely correlated with the laterality index $(r=-0.571 ; p=0.016)$. This study suggests that the degree of shift of activation balance toward the contralesional hemisphere early after stroke increases with the extent of tissue injury and that functional recovery is associated mainly with preservation or restoration of activation in the ipsilesional hemisphere.

Key words: brain ischemia; neuronal plasticity; recovery of function; hemodynamics; magnetic resonance imaging; rats

\section{Introduction}

Stroke is the leading cause of disability in modern society, yet most stroke patients show some, albeit variable, functional recovery over time. This restoration of function is commonly thought to be associated with brain plasticity (Lee and van Donkelaar, 1995; Seil, 1997; Steinberg and Augustine, 1997; Weiller, 1998; Johansson, 2000; Hallett, 2001). Numerous studies have shown that the adult mammalian brain is capable of structural reorganization after injury (Nudo and Friel, 1999). Recent neuroimaging studies have demonstrated changes in brain activation patterns in stroke patients (Weiller, 1998; Cramer and Bastings,

Received May 28, 2002; revised 0ct. 10, 2002; accepted 0ct. 22, 2002.

This study was supported by National Institutes of Health Grants P50-NS10828, R01-HL39810, P01-CA48729, P41-RR14075, R01-NS38477, R01-NS37074, and R01-NS38731. This work was done during the tenure of fellowships from the American Heart Association, New England Affiliate, Inc. (R.M.D., A.B.S.).

Correspondence should be addressed to Dr. Rick M. Dijkhuizen, Image Sciences Institute, University Medical Center Utrecht, Bolognalaan 50, 3584 CJ Utrecht, The Netherlands. E-mail: rick@invivonmr.uu.nl.

Copyright $\odot 2003$ Society for Neuroscience $\quad 0270-6474 / 03 / 230510-08 \$ 15.00 / 0$
2000; Herholz and Heiss, 2000; Rossini and Pauri, 2000; Rijntjes and Weiller, 2002) and in animal stroke models (Reese et al., 2000; Abo et al., 2001; Dijkhuizen et al., 2001; Hoehn et al., 2001; Tuor et al., 2001), yet the spatiotemporal dynamics of reorganization in the brain after stroke and its relationship with tissue status and functional recovery have been incompletely characterized.

Animal stroke models allow reproducible and correlative studies on stroke recovery. With the use of contrast-enhanced functional magnetic resonance imaging (fMRI) in a rat stroke model, we recently described (1) loss of activation in the ipsilesional sensorimotor cortex and (2) a shift of predominantly widespread contralesional responses at $3 \mathrm{~d}$ after stroke to increased perilesional and more restricted contralesional activity at $14 \mathrm{~d}$ after stroke (Dijkhuizen et al., 2001). These results are in agreement with human data describing a relative decrease of contralesional involvement and concentration of ipsilesional activity as a function of time after stroke (Nelles et al., 1999; Marshall et al., 2000). 
A major question that remains unanswered is the functional significance of the pattern of brain reorganization and its relationship to the extent of ischemic damage. In our previous study, which involved a model of permanent focal cerebral ischemia, we were unable to detect statistically significant correlations between the profile of brain activation and the degree of ischemic damage and neurologic dysfunction (Dijkhuizen et al., 2001). Because we used a permanent stroke model, the detection of activationinduced hemodynamic responses with intravascular contrastenhanced fMRI may have been hampered by the chronically reduced perfusion in sensorimotor cortex. Therefore, the goal of the current study was to correlate brain activation patterns with tissue damage, hemodynamics, and neurologic status after transient ischemia. Specifically, we tested the hypotheses that (1) the degree of loss of activation responses in the ipsilesional sensorimotor cortex and a shift in the balance of hemispheric activation are linked to the extent of ischemic damage and (2) functional recovery is correlated with reinstatement of activation in the ipsilesional sensorimotor cortex. Because MRI is a multiparametric neuroimaging tool, supplementary MRI techniques can provide information complementary to the fMRI data. To directly correlate activation-induced responses with perfusion and tissue parameters, MRI measurements of (1) stimulus-induced brain activity, (2) cerebral perfusion, and (3) the transverse relaxation time $\left(\mathrm{T}_{2}\right)$ and (4) diffusion of brain tissue water were performed for each imaging session. Finally, to further elucidate the temporal pattern of brain reorganization, we assessed changes in brain activation patterns from as early as $24 \mathrm{hr}$ up to $14 \mathrm{~d}$ after stroke.

\section{Materials and Methods}

Experimental protocols were institutionally approved in accordance with the NIH Guide for the Care and Use of Laboratory Animals.

Stroke induction. Rats were anesthetized with $1-1.5 \%$ halothane in $\mathrm{N}_{2} \mathrm{O} / \mathrm{O}_{2}$ (70:30) under spontaneous respiration. Body temperature was monitored continuously and maintained at $37^{\circ} \mathrm{C}$ with a thermostatically controlled heating pad. Transient focal cerebral ischemia was induced by $2 \mathrm{hr}$ occlusion of the right middle cerebral artery with an intraluminal filament in adult male Sprague Dawley rats (250-350 gm) (Longa et al., 1989). In brief, a 4.0 nylon monofilament suture coated with silicone was inserted into the external carotid artery. The suture was advanced up the internal carotid artery until mild resistance was felt, indicating that the tip of the filament was properly positioned to occlude blood flow to the middle cerebral artery. After $2 \mathrm{hr}$ of occlusion, the monofilament suture was withdrawn to allow for cerebral reperfusion, and the animal was allowed to recover.

Neurologic examination. At $24 \mathrm{hr}, 3 \mathrm{~d}$, and $14 \mathrm{~d}$ after onset of stroke, the animal's neurologic status was evaluated using the grading system as described by others (Bederson et al., 1986). Grades were given as follows: grade 0 (no neurologic deficit), no observable deficit; grade 1 (moderate neurologic deficit), forelimb flexion; grade 2 (severe neurologic deficit), decreased resistance to lateral push (and forelimb flexion), without circling behavior; grade 3 (severe neurologic deficit), same behavior as grade 2 , with circling.

MRI. In three separate groups of animals, MRI experiments were performed at $1 \mathrm{~d}(n=7)$ (group "day 1 "), $3 \mathrm{~d}(n=7)$ (group "day 3"), and $14 \mathrm{~d}$ after stroke ( $n=7)$ (group "day 14").

Rats were tracheotomized and ventilated mechanically with $1 \%$ halothane in $\mathrm{O}_{2} /$ air (1:1). The right femoral artery was catheterized for monitoring of arterial blood pressure and blood gases. The right femoral vein and jugular vein were cannulated for administration of anesthetic agent and magnetic resonance contrast agent, respectively. Thin copper wires were inserted just underneath the skin on opposite sides of each forelimb at the level of the wrist. Next, rats were paralyzed by an intravenous bolus of pancuronium $(2 \mathrm{mg} / \mathrm{kg})$ followed by continuous infusion (2 $\left.\mathrm{mg} \cdot \mathrm{kg}^{-1} \cdot \mathrm{h}^{-1}\right)$. The anesthetic regimen was changed to a continuous intravenous infusion of $\alpha$-chloralose $\left(40 \mathrm{mg} \cdot \mathrm{kg}^{-1} \cdot \mathrm{h}^{-1}\right)$, preceded by a bolus injection $(50 \mathrm{mg} / \mathrm{kg})$. Stimulation experiments were delayed by at least $1 \mathrm{hr}$ to allow the anesthetic transition.

MRI was done on a $2.0 \mathrm{~T}$ magnet system (Varian Instruments, Palo Alto, CA) using a $3 \mathrm{~cm}$ surface radiofrequency coil that was developed in-house. Body temperature, blood pressure, and blood gases were controlled carefully and maintained at normal values during the MRI experiments.

Multislice spin echo $\mathrm{T}_{2}$ - repetition time $(\mathrm{TR})=2000 \mathrm{msec}$; echo time $(\mathrm{TE})=40,80 \mathrm{msec}]$ and diffusion-weighted images $(\mathrm{TR} / \mathrm{TE}=2000 / 40$ msec; $b=150,850,1550 \mathrm{sec} / \mathrm{mm}^{2}$; diffusion-encoding gradients in three directions) were acquired [field-of-view (FOV) $=25 \times 25 \mathrm{~mm}^{2} ; 64 \times 64$ data matrix; nine $1.5 \mathrm{~mm}$ slices], from which we calculated twodimensional maps of the $\mathrm{T}_{2}$ and the mean trace of the apparent diffusion coefficient (ADC) of tissue water, respectively (van Gelderen et al., 1994; Loubinoux et al., 1997). Second, boluses of the intravascular contrast agent monocrystalline iron oxide nanocolloid (MION) (up to a total amount of $10-15 \mathrm{mg} / \mathrm{kg}$ ), injected through the jugular vein, were combined with single-slice dynamic susceptibility contrast-enhanced MRI [single-shot gradient recalled echo planar imaging (EPI); TR/TE = $175 / 22$ msec; FOV $=25 \times 25 \mathrm{~mm}^{2} ; 32 \times 32$ data matrix, zero-filled to $64 \times 64$; slice thickness $1.5 \mathrm{~mm} ; 500$ consecutive images]. The selected slice position was at $\sim 1 \mathrm{~mm}$ posterior to bregma according to Paxinos and Watson (1997) and matched with its corresponding slice in the multislice MRI data sets. Relative cerebral blood flow index $\left(\mathrm{CBF}_{\mathrm{i}}\right)$ values were obtained for each voxel as described by Østergaard et al. (1996). Arterial input data were obtained directly from the susceptibility contrast-enhanced images in two voxels in the left and right internal carotid artery.

Multislice contrast-enhanced MRI (single-shot gradient recalled EPI; $\mathrm{TR} / \mathrm{TE}=2000 / 22 \mathrm{msec} ; 510$ time points; FOV $=25 \times 25 \mathrm{~mm}^{2} ; 32 \times 32$ data matrix, zero-filled to $64 \times 64$; nine $1.5 \mathrm{~mm}$ slices) was performed after the injections of MION. Cerebral blood volume (CBV)-weighted images were calculated as described previously (Hamberg et al., 1996; Mandeville et al., 1998). Local relative CBV in specific regions-of-interest (ROIs) (see below) before the stimulation paradigm was expressed as a percentage of contralateral. Dynamic activation-induced CBV changes were measured by acquiring contrast-enhanced functional MR images during electrical stimulation ( $5 \mathrm{~V}$ for $0.5 \mathrm{msec}$ at $3 \mathrm{~Hz}$ during $40 \mathrm{sec}$ ) to first the right and then (after $40 \mathrm{sec}$ ) the left forelimb (six stimulation on-off periods per forelimb, starting and ending with a stimulation off period) (total acquisition time, $17 \mathrm{~min}$ ). Functional activation maps were generated by a voxel-wise $t$ test between the stimulated and nonstimulated conditions. $p<0.05$ with Bonferroni correction was used as the statistical threshold for significant activation response.

Cerebrovascular reserve capacity was assessed by acquiring CBVweighted images as described above, during $10 \mathrm{~min}$ inhalation of $5 \% \mathrm{CO}_{2}$ in balanced $\mathrm{O}_{2}$ (after 10 min of breathing with $100 \% \mathrm{O}_{2}$ ). We characterized the plateau $\mathrm{CO}_{2}$-induced $\mathrm{CBV}$ change $\left(\Delta \mathrm{CBV}_{\max }\left[\mathrm{CO}_{2}\right]\right)$ as the mean $\mathrm{CBV}$ change between 2 and $10 \mathrm{~min}$ after onset of $5 \% \mathrm{CO}_{2}$ inhalation.

Data analysis. Lesion volumes were calculated from the multislice $\mathrm{T}_{2}$ datasets with use of the image analysis software package Alice (Hayden Image Processing Group, Boulder, $\mathrm{CO}$ ). $\mathrm{T}_{2}$ lesion volume was defined as the ipsilateral parenchymal brain volume with $\mathrm{T}_{2}$ values higher than the mean $+2 \mathrm{SD}$ of the $\mathrm{T}_{2}$ in contralateral tissue.

The total area of significant forelimb stimulation-induced CBV responses was calculated in each hemisphere with Alice. The laterality index, which expresses relative activity in the contralateral hemisphere as compared with the ipsilateral hemisphere, was defined as $(C-I) /(C+$ $I)$, where $C$ and $I$ are the total activation volumes in the hemisphere contralateral and ipsilateral to the stimulated forelimb, respectively. The time course of CBV changes was averaged over the six stimulation on-off periods for each animal. From this averaged time course we calculated the mean activation-induced CBV change during the last $10 \mathrm{sec}$ of stimulation for both forelimbs of each individual $\left(\Delta \mathrm{CBV}_{\max }[\right.$ act.] $)$, which was used as an index for the degree of activation (Dijkhuizen et al., 2001).

ROI analyses were performed on MRI parameters (i.e., $\mathrm{T}_{2}, \mathrm{ADC}, \mathrm{CBF}_{\mathrm{i}}$, $\mathrm{CBV}, \Delta \mathrm{CBV}_{\max }\left[\mathrm{CO}_{2}\right]$, and $\Delta \mathrm{CBV}_{\max }[$ act.]) by positioning ROIs [five voxels $\left.\left(1.14 \mathrm{~mm}^{3}\right)\right](1)$ in the area of most significant activation during 
Table 1. Neurologic scores and $\mathrm{T}_{2}$ lesion volumes at 1, 3, and $14 \mathrm{~d}$ after $2 \mathrm{hr}$ middle cerebral artery occlusion

\begin{tabular}{lllll}
\hline \multirow{3}{*}{ Group } & \multicolumn{2}{l}{ Neurologic score } & \\
\cline { 2 - 3 } & Day 1 & Day 3 & Day 14 & \\
\hline Day 1 & $2.0 \pm 0.9$ & & $T_{2}$ lesion volume $\left(\mathrm{mm}^{3}\right)$ \\
Day 3 & $2.2 \pm 0.4$ & $2.0 \pm 0.7$ & & $151 \pm 57$ \\
Day 14 & $2.3 \pm 0.8$ & $1.6 \pm 0.5$ & $1.0 \pm 0.6^{*}$ & $119 \pm 92$ \\
\hline${ }^{*} p<0.05$ versus day 1. & & &
\end{tabular}

stimulation of the unimpaired, right forelimb [i.e., the contralesional forelimb region of the primary somatosensory cortex ( $\mathrm{S} 1 \mathrm{fl}$ ) and primary motor cortex (M1)] (Dijkhuizen et al., 2001), (2) in the homologous contralateral region (i.e., ipsilesional $\mathrm{M} 1 / \mathrm{S} 1 \mathrm{fl}$ ), and (3) in the ischemic core (i.e., the ipsilesional parietal cortex). Note that the ROI in ipsilesional M1/S1fl may represent different degrees of ischemic damage across individual animals, because in this animal stroke model M1/S1fl typically lies at the border of the ischemic lesion and becomes more or less affected depending on the severity of ischemia.

All values are expressed as mean $\pm \mathrm{SD}$. Statistical comparisons were performed using one-way ANOVA (with repeated measures where appropriate) with post hoc Tukey test for multiple comparisons and paired Student's $t$ test. For correlation analyses we applied the Pearson product moment correlation test (and linear regression) or the Spearman rank order correlation test (and ordinal logistic regression). To separate the possible confounding effects of the time after stroke from any independent relationship of laterality index and neurologic status, we used multiple linear and ordinal logistic modeling. $p$ values of $<0.05$ were considered significant.

\section{Results}

\section{Neurologic status}

Animals demonstrated neurologic deficits at all time points after stroke; however, functional scores significantly improved over $14 \mathrm{~d}$ (Table 1).

\section{Ischemic damage}

MRI revealed unilateral lesions in the middle cerebral artery territory, generally involving the lateral cortex and striatum (Fig. 1). Lesions were characterized by a reduced ADC and increased $\mathrm{T}_{2}$ (Fig. 1, top panel). Because our study involved a model of transient occlusion of the middle cerebral artery, ischemia was followed by reperfusion. In fact, we detected moderate hyperperfusion at $24 \mathrm{hr}$ after stroke (Fig. 1, top panel). After $3 \mathrm{~d}$, ADC values had pseudonormalized in large parts of the lesion (Fig. 1, middle panel). $\mathrm{T}_{2}$ and perfusion parameters continued to be high as compared with contralateral. At day 14, there was mild hyperperfusion in the lesion, and $\mathrm{ADC}$ and $\mathrm{T}_{2}$ values were elevated (Fig. 1, bottom panel ). $\mathrm{T}_{2}$ lesion volumes are given in Table 1 . Depending on the extent of the lesion, $\mathrm{ADC}, \mathrm{T}_{2}$, and perfusion were mildly to severely affected in the forelimb region of the sensorimotor cortex (i.e., M1/S1fl) in the ipsilesional hemisphere.

\section{Cerebrovascular reactivity}

To test cerebrovascular reactivity, we applied a $\mathrm{CO}_{2}$ challenge and measured CBV changes in the brain. Results are shown in Figure 2. Inhalation of $5 \% \mathrm{CO}_{2}$ raised the arterial $\mathrm{P}_{\mathrm{CO}_{2}}$ from $37.0 \pm 2.3$ to $53.6 \pm 5.1 \mathrm{mmHg}$, resulting in a clear CBV increase in the contralesional hemisphere. At $1 \mathrm{~d}$ after transient focal ischemia, the $\mathrm{CO}_{2}$-induced $\mathrm{CBV}$ response was lowered significantly throughout most of the ipsilesional hemisphere. After $3 \mathrm{~d}$, we found an enhanced CBV response in the ipsilesional parietal cortex. In M1/S1fl, at the border of the lesion, CBV changes were not significantly different as compared with contralesional. After $14 \mathrm{~d}, \mathrm{CO}_{2}$-induced $\mathrm{CBV}$ changes were subnormal in the ipsile-
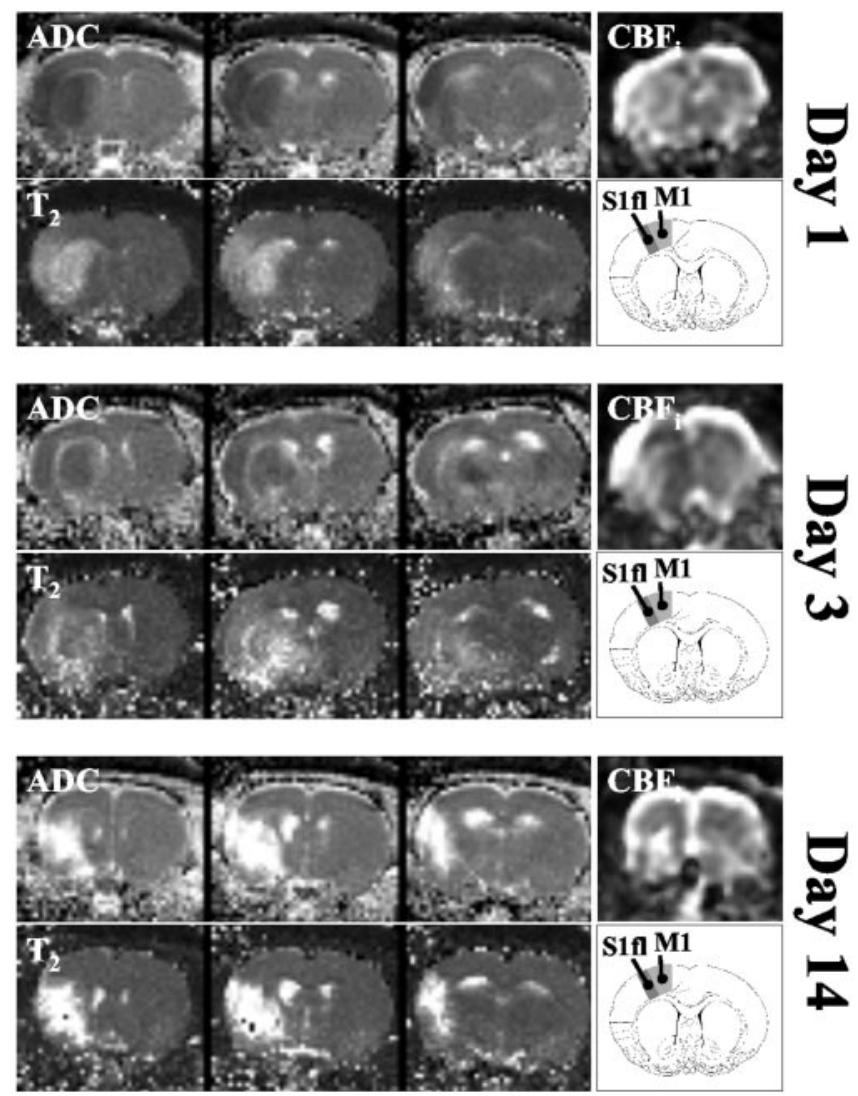

Figure 1. Multislice $A D C$ and $T_{2}$ maps (3 adjacent slices are shown) and single-slice $C B F_{i}$ maps (same slice position as the left image of the $A D$ (and $\mathrm{T}_{2}$ maps) at $1 \mathrm{~d}$ (top panel), $3 \mathrm{~d}$ (middle panel), and $14 \mathrm{~d}$ (bottom panel) after $2 \mathrm{hr}$ middle cerebral artery occlusion. Each panel represents data from a single animal. Below the $\mathrm{CBF}_{\mathrm{i}}$ maps is an outline of a coronal rat brain section centered $0.7 \mathrm{~mm}$ from bregma [reproduced from Paxinos and Watson (1997), with permission from Academic Press]. S1fl, Forelimb region of the primary somatosensory cortex; M1, primary motor cortex (Paxinos and Watson, 1997).

sional hemisphere, but this was not significant for M1/S1fl. Correlation analyses did not show any significant relationship between $\mathrm{CO}_{2}$-induced $\Delta \mathrm{CBV}_{\text {max }}\left[\mathrm{CO}_{2}\right]$ and tissue or perfusion parameters.

\section{Cerebral activation responses}

Figure 3 shows $\mathrm{T}_{2}$-weighted images overlaid by statistical activation maps and graphs of the time course of CBV changes in $\mathrm{M} 1 / \mathrm{S} 1 \mathrm{fl}$ for the different animal groups. Images, maps, and graphs represent data averaged across animals. Stimulation of the unimpaired forelimb invariably resulted in a significant activation-induced CBV response in $\mathrm{M} 1 / \mathrm{S} 1 \mathrm{fl}$ in the unaffected, contralesional hemisphere (i.e., contralateral to the stimulated limb) (Fig. 3). The stereotaxic coordinates of the center of the focus of activation on stimulation of the unimpaired limb were $0.1 \pm 1.1 \mathrm{~mm}$ anterior, $3.7 \pm 0.2 \mathrm{~mm}$ lateral, and $1.7 \pm 0.3 \mathrm{~mm}$ ventral to bregma (averaged over all 21 animals), which corresponds to S1fl according to Paxinos and Watson (1997). In 8 of the total group of 21 animals, unilateral or bilateral responses were also detected in the thalamus. Stimulation of the impaired forelimb at days 1 and 3 was accompanied by a diminished amplitude of the activation-induced CBV response in ipsilesional M1/S1fl (Fig. 3). Nevertheless, small but significant responses were detected in the ipsilesional hemisphere, both in and remote (anteriorly and posteriorly) from M1/S1fl (the mean stereotaxic 

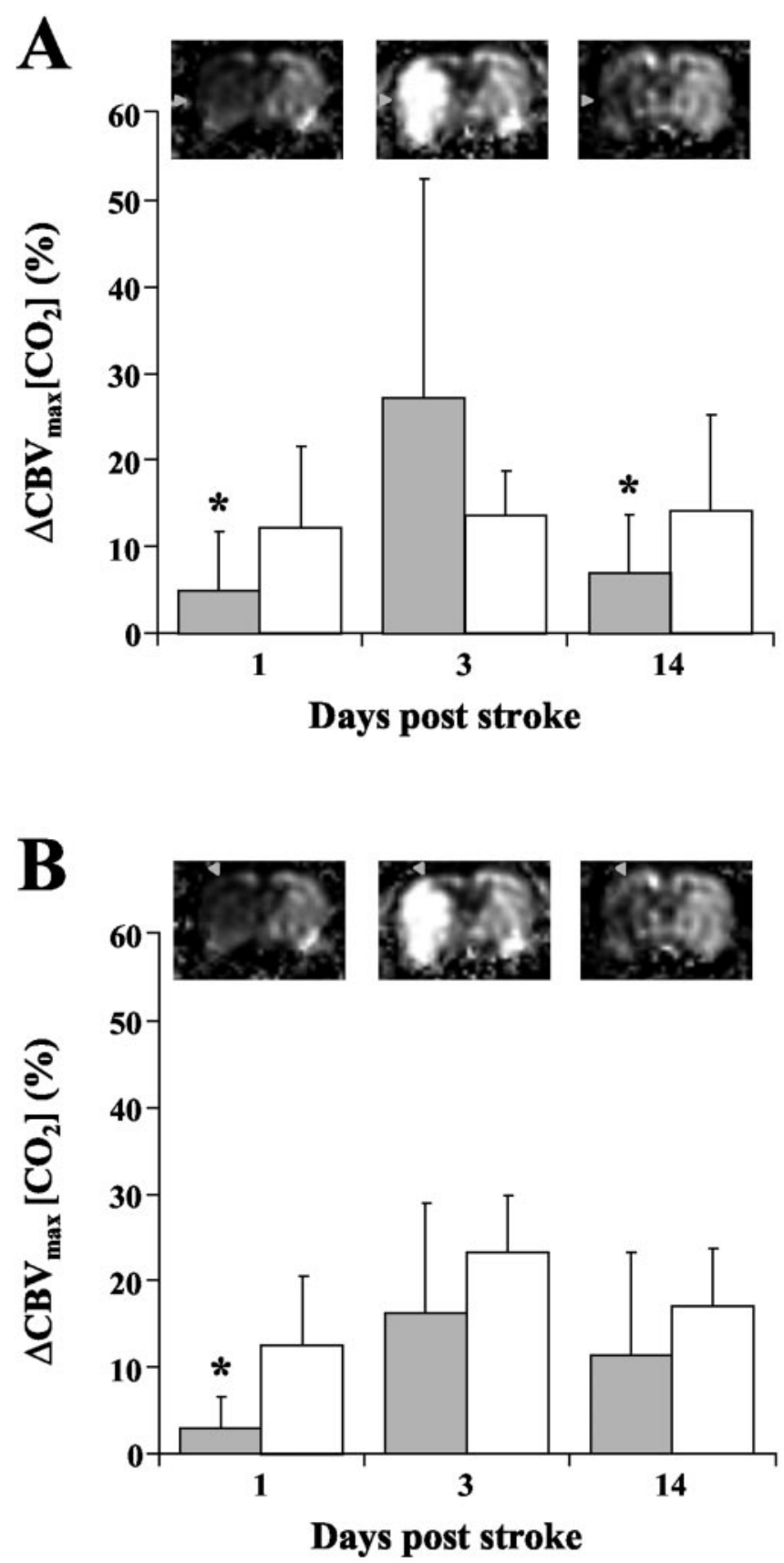

Figure 2. Plateau CBV change $\left(\Delta \mathrm{CBV}_{\max }\left[\mathrm{CO}_{2}\right]\right)$ in the ipsilesional ( gray bars) and contralesional (white bars) parietal cortex $(A)$ and $\mathrm{M} 1 / \mathrm{S} 1 \mathrm{fl}(B)$ during $5 \% \mathrm{CO}_{2}$ inhalation at 1,3 , and $14 \mathrm{~d}$ after $2 \mathrm{hr}$ middle cerebral artery occlusion (mean $+\mathrm{SD} ; n=7$ ). ${ }^{*} p<0.05$ versus contralesional. Insets show averaged $\Delta \mathrm{CBV}_{\max }\left[\mathrm{CO}_{2}\right]$ maps of a coronal rat brain slice at the specific time points after stroke (data are averaged across animals). Gray arrowheads point to ROl locations in the parietal cortex $(A)$ and $\mathrm{M} 1 / \mathrm{S} 1 \mathrm{fl}(B)$.

coordinates were $-1.5 \pm 2.5 \mathrm{~mm}$ anterior, $3.6 \pm 0.7 \mathrm{~mm}$ lateral, and $1.7 \pm 0.4$ ventral to bregma at day 1 , and $-1.3 \pm 2.3 \mathrm{~mm}$ anterior, $4.0 \pm 0.4 \mathrm{~mm}$ lateral, and $1.9 \pm 0.6$ ventral to bregma at day 3). Extensive activation-induced responses in the contralesional hemisphere (i.e., ipsilateral to the stimulated limb), involving large parts of the neocortex, were found in five of seven animals in both the $24 \mathrm{hr}$ and $3 \mathrm{~d}$ post-stroke groups, respectively (Fig. 3). Correspondingly, the laterality index, which describes the degree of asymmetry in activation between the hemispheres, was considerably reduced at these time points (Fig. 4). After $14 \mathrm{~d}$, the focus of activation had returned to ipsilesional M1/S1fl (the mean stereotaxic coordinates were $-1.0 \pm 0.9 \mathrm{~mm}$ anterior, $4.1 \pm 0.2 \mathrm{~mm}$ lateral, and $2.0 \pm 0.4$ ventral to bregma at day 14) (Fig. 3). At this stage, signs of activation during impaired forelimb stimulation were mostly absent in the contralesional hemisphere, and the laterality index was not significantly different from the laterality index during unimpaired forelimb stimulation (Fig. 4). Significant unilateral or bilateral thalamic responses on stimulation of the impaired forelimb were detected in three, one, and two animals in the day 1, 3 and 14 groups, respectively.

\section{Correlation analyses}

Correlation analyses demonstrated that changes in activation patterns were related to extent of ischemic damage. We found significant relationships between lesion size and impaired forelimb stimulation-induced $\Delta \mathrm{CBV}_{\max }$ [act.] in ipsilesional M1/S1fl at days 3 and 14 (Fig. 5A). The laterality index was correlated negatively with lesion size in the day 1 group (Fig. $5 B$ ). The main findings from the correlation analyses are summarized in Table 2. Significant relationships between the laterality index and $\mathrm{T}_{2}$ and ADC in ipsilesional M1/S1fl were evident at days 1 and 14. We did not find a significant correlation between perfusion status (i.e., $\mathrm{CBV}$ and $\mathrm{CBF}_{\mathrm{i}}$ ) and impaired forelimb stimulation-induced $\Delta \mathrm{CBV}_{\max }$ [act.] in ipsilesional $\mathrm{M} 1 / \mathrm{S} 1 \mathrm{fl}$ or the laterality index (data not shown). There were no significant relationships between activation-induced $\Delta \mathrm{CBV}_{\max }$ [act.] during stimulation of the impaired forelimb and $\mathrm{CO}_{2}$-induced $\Delta \mathrm{CBV}_{\max }\left[\mathrm{CO}_{2}\right]$ in ipsilesional M1/S1fl (data not shown). Finally, we found no significant correlations between neurologic status and impaired forelimb stimulation-induced $\Delta \mathrm{CBV}_{\max }$ [act.] in ipsilesional $\mathrm{M} 1 / \mathrm{S} 1 \mathrm{fl}$ or the laterality index. However, when all time points were grouped together, neurologic status was inversely correlated with the laterality index $(r=-0.571 ; p=0.016)$. Because both laterality index and neurologic status were correlated with the time after stroke, we included the time in the model. The overall model was significant $\left(r^{2}=0.371 ; p=0.039\right)$. However, neither laterality index nor the time from the stroke demonstrated a significant independent relationship with neurologic score $(p=0.305$ and $p=0.159$, respectively). Thus, laterality index and neurologic status appear to change in parallel over time.

\section{Discussion}

Both ipsilesional and contralesional brain reorganization are known to occur after stroke. The main finding of this study is that the degree of shift of activation balance toward the contralesional hemisphere early after stroke increases with the extent of tissue injury and that functional recovery is associated mainly with preservation or restoration of activation in the ipsilesional hemisphere.

We assessed spatiotemporal characteristics of changes in brain activation patterns in relation to tissue, perfusion, and neurologic status after transient focal cerebral ischemia in rats. With the use of contrast-enhanced CBV-weighted fMRI, we detected distinct activation-induced responses in the contralesional hemisphere after stimulation of the impaired forelimb early after transient ischemia, which is in agreement with two previous studies in models of permanent cerebral ischemia (Abo et al., 2001; Dijkhuizen et al., 2001). Importantly, this contralesional activity was present as early as $24 \mathrm{hr}$ and remained evident at $3 \mathrm{~d}$ after stroke. Responses in ipsilesional M1/S1fl were diminished at these stages. As a result, laterality indices were reduced significantly. Chronically after stroke, activity in ipsilesional M1/S1fl 


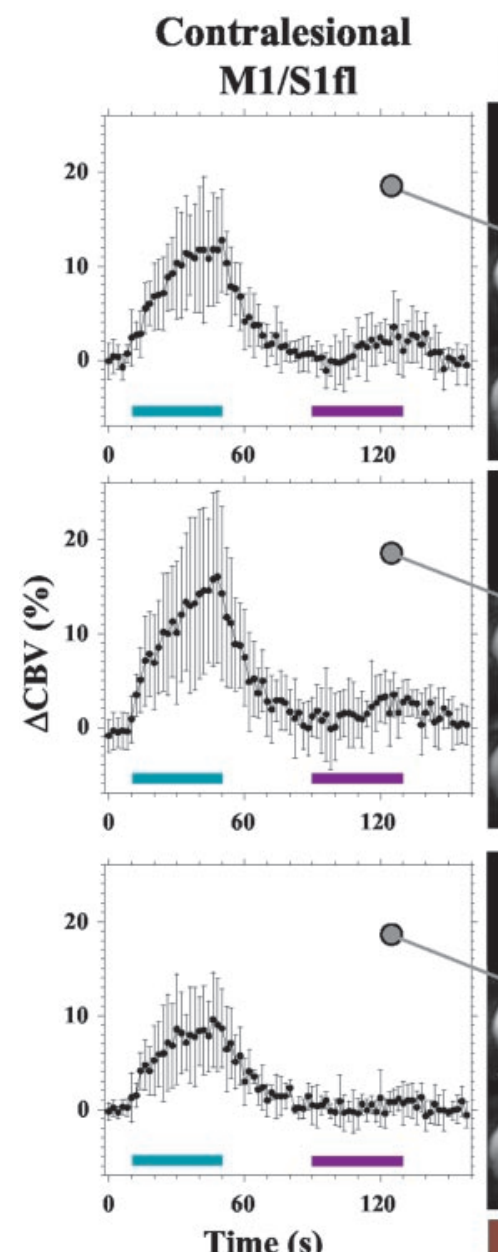

Unimpaired limb
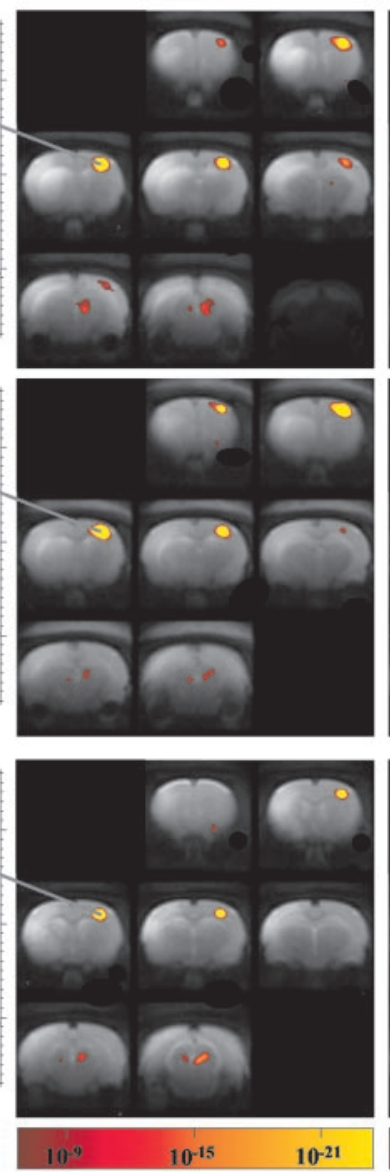
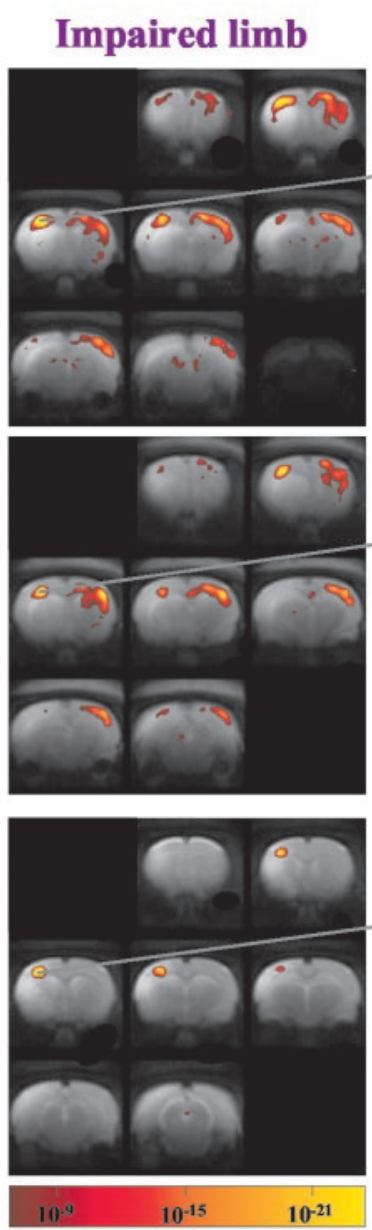
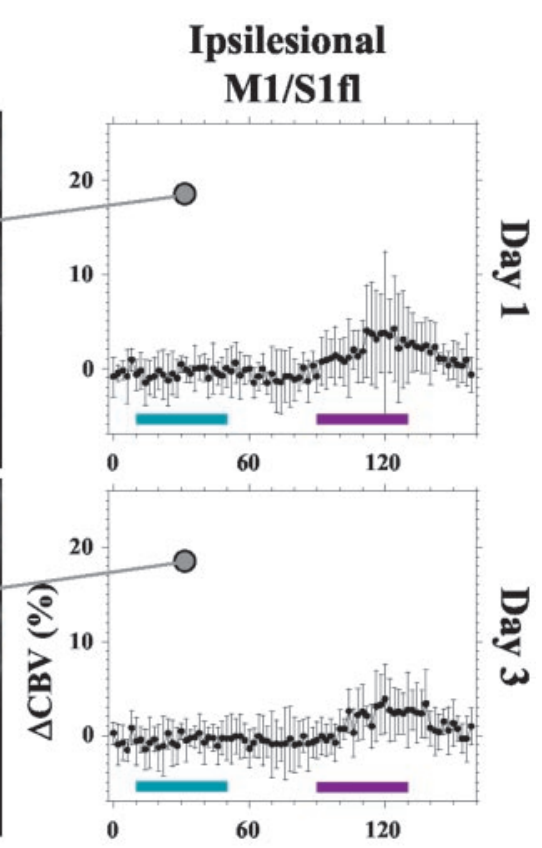

0

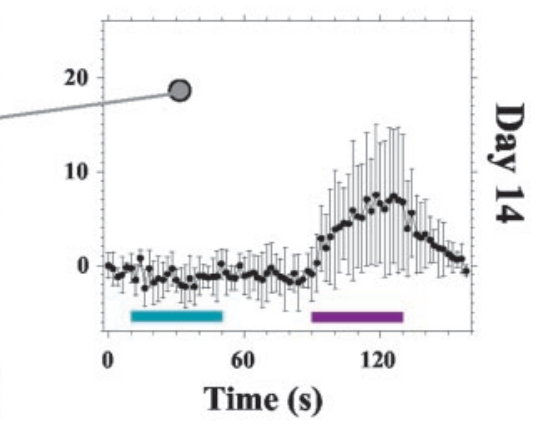

Figure 3. Averaged $\mathrm{T}_{2}$-weighted images of coronal rat brain slices overlaid by statistical activation maps, calculated from the averaged activation-induced cerebral CBV changes ( $n=7$ ) (data are averaged across animals). The map of $p$ values has been color-coded corresponding to the degree of significance (see bars below images). The graphs show the mean of the averaged time course of CBV changes (averaged across six on - off periods for each forelimb) in an ROI (5 voxels) in ipsilesional (right) and contralesional (left) M1/S1fl (mean \pm SD; $n=7$ ). Top row, 24 hr after stroke; middle row, 3 dafter stroke; bottom row, $14 \mathrm{~d}$ after stroke. Right, Unimpaired forelimb stimulation (represented by the green bars in the graphs) induced significant activation responses in the contralateral (right) M1/S1fl at all time points. Stimulation of the left, impaired forelimb (represented by the blue bars in the graphs) resulted in diminished responsiveness in the right, ipsilesional M1/S1fl at 24 $\mathrm{hr}$ and $3 \mathrm{~d}$ after stroke. However, clear responses were found in the contralesional hemisphere. After $14 \mathrm{~d}$, activation responses were predominantly in ipsilesional M1/S1fl. Infarction areas are characterized by increased $\mathrm{T}_{2}$-weighted signal intensity.

had recovered, contralesional responses were minor, and laterality indices were close to control values.

\section{Correlation between loss of activation responses and ischemic damage}

An important goal of this study was to correlate the pattern of brain reorganization with ischemic damage. Brain tissue status was assessed with $\mathrm{T}_{2}$ - and diffusion-weighted MRI. Brain ADC values that are reduced acutely after stroke reflect cytotoxic edematous tissue (Moseley et al., 1990; Verheul et al., 1994), whereas increases in $\mathrm{T}_{2}$ and $\mathrm{ADC}$ are associated with the development of vasogenic edema and tissue degeneration (Pierpaoli et al., 1993; Matsumoto et al., 1995). $\mathrm{T}_{2}$ maps were used to calculate lesion volumes, because $\mathrm{T}_{2}$ changes are unidirectional and correlate with irreversible tissue damage (Allegrini and Sauer, 1992; Palmer et al., 2001). We found an inverse relationship between lesion size and the activation-induced hemodynamic response in ipsilesional M1/S1fl during stimulation of the impaired forelimb, as characterized by $\Delta \mathrm{CBV}_{\max }$ [act.], at days 3 and 14 . The middle cerebral artery supplies various brain regions that are part of the sensorimotor network. Large lesions in the middle cerebral artery territory will therefore affect a great portion of projections to and from M1/S1fl, resulting in loss of neuronal output. Decreased activation responses could be the direct result of injury to M1/ S1fl; however, loss of activation may not necessarily be linked primarily to focal damage in this area, because impaired forelimb stimulation-induced $\Delta \mathrm{CBV}_{\max }$ [act.] in ipsilesional M1/S1fl correlated more strongly with $\mathrm{T}_{2}$ lesion size than with local $\mathrm{T}_{2}$ (or $\mathrm{ADC}$ ) in this area. A long-lasting synaptic transmission defect has been detected in the sensorimotor cortex after transient middle cerebral artery occlusions in rats (Bolay and Dalkara, 1998) that may occur without apparent histological damage (Aoyagi et al., 1998). Importantly, it has been shown that impaired excitatory neurotransmission after temporary ischemia has the potential to recover (Aoyagi et al., 1998), which could account for reinstatement of activation as seen after $14 \mathrm{~d}$ in our study.

In addition to the brain tissue status, we calculated relative $\mathrm{CBF}_{\mathrm{i}}$ and $\mathrm{CBV}$ with the use of dynamic and steady-state susceptibility contrast-enhanced MRI, respectively. Because we did not detect reduced perfusion levels at the examined time points (in fact, $2 \mathrm{hr}$ middle cerebral artery occlusion in the rat was followed by chronic hyperperfusion), loss of detection of fMRI signals 


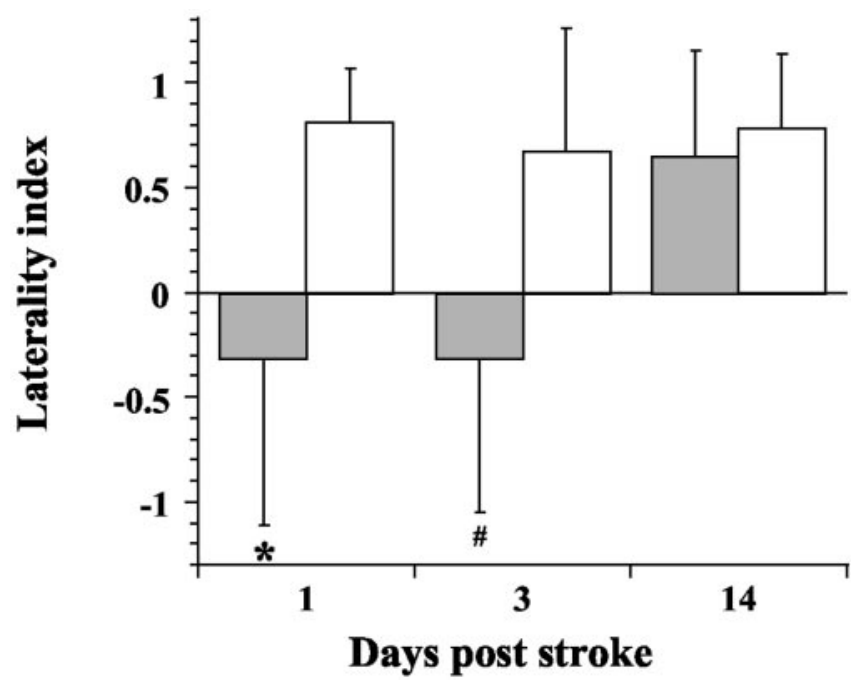

Figure 4. Laterality indices during stimulation of the left, impaired ( gray bars) and right, unimpaired forelimb (white bars) at 1, 3, and $14 \mathrm{~d}$ after $2 \mathrm{hr}$ middle cerebral artery occlusion (mean $\pm S D ; n=7$ ). The negative laterality indices during impaired forelimb stimulation at days 1 and 3 represent the enhanced relative activity in the contralesional hemisphere (i.e., ipsilateral to the stimulated forelimb) as compared with the ipsilesional hemisphere. At $14 \mathrm{~d}$ after stroke, the laterality index was near baseline, indicating that bulk activation was in the ipsilesional hemisphere (i.e., contralateral to the stimulated forelimb). ${ }^{*} p<0.05 ;{ }^{*} p=0.052$.

cannot be explained by insufficient distribution of the contrast agent. Still, altered CBF and CBV dynamics after temporary stroke may have affected the fMRI signal. Nevertheless, we did not detect a significant relationship between activation-induced $\Delta \mathrm{CBV}_{\max }$ [act.] during stimulation of the impaired forelimb and perfusion parameters in ipsilesional M1/S1fl. Defective cerebrovascular reactivity may be involved in loss of fMRI-detected activation. There was, however, no significant relationship between activation-induced and $\mathrm{CO}_{2}$-induced $\Delta \mathrm{CBV}_{\max }$. This is in agreement with a study in patients with chronic major cerebral arterial steno-occlusive lesions, in which motor task-induced CBF increase in the primary sensorimotor cortex was not seriously affected by hemodynamic insufficiency (characterized by abolished response to the vasodilator acetazolamide) (Inao et al., 1998). Thus, it appears that loss of activation responses in M1/S1fl cannot be consistently explained by deficient vasoreactivity and is more related to damage to the sensorimotor network. Nevertheless, both factors play a role, and their contribution may vary under certain conditions. In contrast to days 3 and 14, no significant correlation between impaired forelimb stimulationinduced $\triangle \mathrm{CBV}_{\max }$ [act.] and ischemic damage in ipsilesional M1/ S1fl was found at day 1 , and loss of cerebrovascular reactivity was strongest at this acute stage.

\section{Correlation between brain reorganization and ischemic damage}

Along with loss of activation responses in ipsilesional M1/S1fl, stroke led to augmented responsiveness in the contralesional hemisphere, characterized by a reduction of the laterality index. Enhanced contralesional activation has been described in earlier studies in stroke patients and animal models and appears to be strongest early after stroke (Cuadrado et al., 1999; Marshall et al., 2000; Dijkhuizen et al., 2001) (however, see also Calautti et al. 2001). At later stages, the involvement of the ipsilesional hemisphere improves and the laterality index normalizes.

We found significant correlations between the laterality index

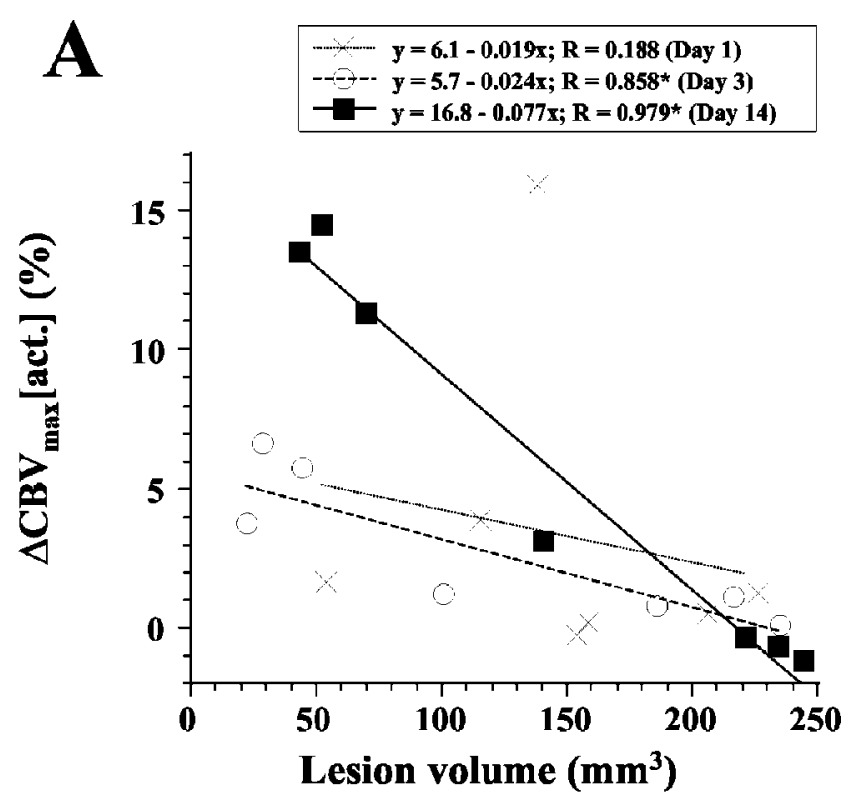

B

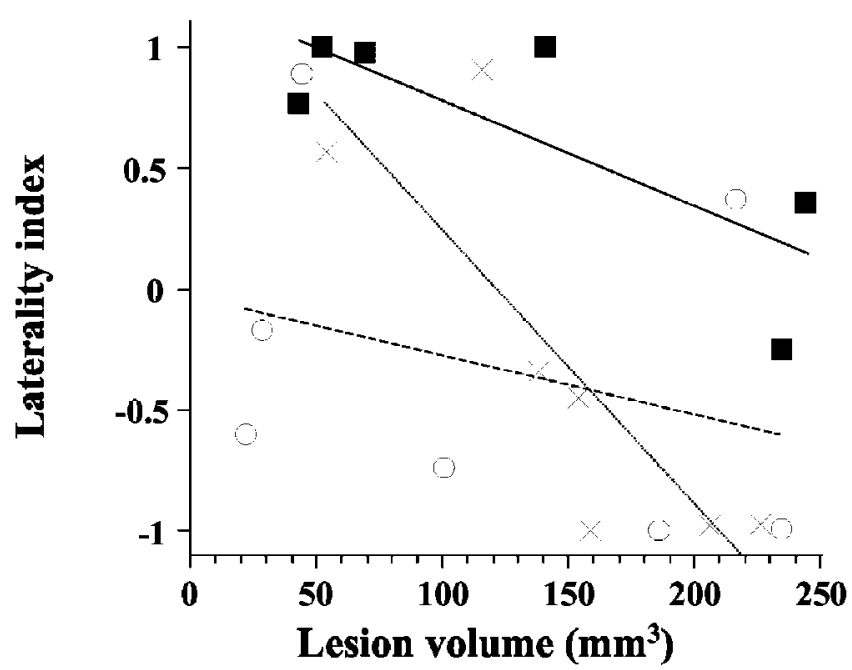

Figure 5. Relationship between lesion volume and plateau $C B V$ change $\left(\Delta \mathrm{CBV}_{\max }[\mathrm{act}].\right)$ in ipsilesional M1/S1fl $(A)$ and laterality index $(B)$ during impaired forelimb stimulation at $1 \mathrm{~d}$ $(\times), 3 \mathrm{~d}(\bigcirc)$, and $14 \mathrm{~d}(\boldsymbol{\square})$ after $2 \mathrm{hr}$ middle cerebral artery occlusion ( $n=7$ for each time point). ${ }^{*} p<0.05$

and the degree of brain injury. At day 1 the laterality index was correlated negatively with lesion size (there was a trend toward a significant relationship at day 14). Also, the laterality index was correlated with the degree of tissue damage in M1/S1fl, expressed by the local $\mathrm{ADC}$ and $\mathrm{T}_{2}$, at days 1 and 14 after stroke. The lack of correlation between the laterality index and the $\mathrm{ADC}$ and $\mathrm{T}_{2}$ at $3 \mathrm{~d}$ after stroke is most likely attributable to the pseudonormalization of $\mathrm{ADC}$ and partial recovery of $\mathrm{T}_{2}$ values at subacute stages, as a result of cellular lysis (Pierpaoli et al., 1993) and transient decline of tissue water content (Lin et al., 2002), respectively. Speculatively, consequent release of tissue pressure may also facilitate expansion of intact blood vessels, which could explain the noteworthy enhanced vasoreactivity to hypercapnia at this stage. 
Table 2. Correlation between activation parameters (impaired forelimb stimulation-induced $\Delta \mathrm{CBV}_{\text {max }}$ [act.] in ipsilesional $\mathrm{M1} / \mathrm{S} 1 \mathrm{fl}$; laterality index) and cerebral tissue injury (ADC and $\mathrm{T}_{2}$ in ipsilesional M1/S1fl; lesion size) and neurologic status (neurologic score) at 1 (1d), 3 (3d), and $14 \mathrm{~d}$ after stroke (14d)

\begin{tabular}{|c|c|c|c|c|c|c|}
\hline \multirow[b]{2}{*}{ Parameter } & \multicolumn{3}{|c|}{$\Delta$ CBV $_{\text {max }}$ [act.] } & \multicolumn{3}{|c|}{ Laterality index } \\
\hline & $1 d$ & $3 d$ & $14 d$ & $1 d$ & $3 d$ & $14 d$ \\
\hline$A D C$ & 0.524 & 0.329 & -0.721 & $0.782^{*}$ & 0.192 & $-0.970^{*}$ \\
\hline $\mathrm{T}_{2}$ & -0.508 & $-0.795^{*}$ & -0.678 & $-0.807^{*}$ & -0.337 & $-0.898^{*}$ \\
\hline Lesion size & -0.188 & $-0.858^{*}$ & $-0.979^{*}$ & $-0.826^{*}$ & -0.313 & -0.792 \\
\hline Neurologic score & -0.359 & -0.224 & -0.535 & -0.239 & -0.671 & -0.343 \\
\hline
\end{tabular}

Results are expressed by correlation coefficient values, $r$. All values are from parametric correlations, except for correlations with neurologic score, which were nonparametric.

${ }^{*} p<0.05$.

The correlation between brain reorganization and ischemic tissue damage is in agreement with a recent fMRI study in patients with cerebral autosomal dominant arteriopathy with subcortical infarcts and leukoencephalopathy, in which the motor cortex laterality index correlated with the relative $N$-acetylaspartate decrease in white matter, which was used as an index of axonal injury (Reddy et al., 2002). Hyperexcitability after stroke is likely to be the result of disinhibition or potentiation of existing neuronal circuitry, involving GABAergic and glutamatergic pathways (Witte et al., 2000). Downregulation of $\mathrm{GABA}_{\mathrm{A}}$ receptor binding and an increase of NMDA receptors have been found in the contralesional hemisphere and in perilesional areas as early as $4 \mathrm{hr}$ after experimental stroke (Que et al., 1999a,b). In the current study, widespread contralesional activity, and occasionally perilesional responses, were evident within $24 \mathrm{hr}$ after ischemia, which emphasizes the rapidity of these plastic changes in the brain. Anatomic remodeling, e.g., dendritic outgrowth and synaptogenesis, has also been found in areas remote from a cerebral lesion (Jones and Schallert, 1992; Stroemer et al., 1995; Jones et al., 1996; Kawamata et al., 1997). However, these processes may require days to weeks to develop (Jones and Schallert, 1992; Jones et al., 1996) and may be more involved in reinstatement of activation in the ipsilesional M1/S1fl and chronic perilesional activity.

\section{Correlation between brain reorganization and functional status}

From the above it appears clear that changes in the pattern of activation are linked directly to the degree of ischemic injury. However, an important question is how cerebral reorganization is associated with functional recovery. Despite a significant negative relationship between laterality index and neurologic status when all data were grouped together, no significant correlations were found at the individual time points. The lack of a significant effect that was independent of the time after stroke prohibits any presumption of a causal relationship between a shift of sensorimotor activation patterns and restoration of forelimb function. Moreover, it should be mentioned that the behavioral test that we used provided an overall score of neurologic function and did not inform exclusively on sensorimotor function of the forelimb. A stronger relationship between brain reorganization and functional recovery may have been found when neural and functional measures were matched more specifically. Nonetheless, the trend of a negative association between laterality index and neurologic status is in line with earlier findings by others (Marshall et al., 2000) and by us (Dijkhuizen et al., 2001) that restoration of sensorimotor function is paralleled by recovery of the laterality index, and it is in agreement with a transcranial magnetic stimulation study that described that increased excitability in the contralesional hemisphere was found predominantly in poorly recovered subjects (Netz et al., 1997). In addition, the functional significance of these reorganizational alterations is emphasized further by a rehabilitation study that showed a shift of activation from predominantly contralesional to a more balanced ipsilesional and contralesional pattern, in parallel with improved recovery (Liepert et al., 2000).

\section{Concluding remarks}

This study shows that the spatiotemporal pattern of shifts in cerebral activation after stroke is closely related to the degree of ischemic damage. Our results provide correlative evidence that good neurologic function is associated with a "normal" balance of activation in the cerebral hemispheres. Functional recovery seems to depend predominantly on preservation, reinstatement, or intrahemispheric shift of activation within the ipsilesional hemisphere. Although functional fields in the contralesional hemisphere may be involved in upholding and restoring function, acute contralesional activity appears to be mainly a direct response to the ischemic injury, without a clear functional significance. Finally, our results suggest that the application of fMRI in stroke patients can provide important prognostic information on functional outcome. This may aid in the selection of therapeutic strategies that could improve functional recovery after stroke.

\section{References}

Abo M, Chen Z, Lai LJ, Reese T, Bjelke B (2001) Functional recovery after brain lesion-contralateral neuromodulation: an fMRI study. NeuroReport 12:1543-1547.

Allegrini PR, Sauer D (1992) Application of magnetic resonance imaging to the measurement of neurodegeneration in rat brain: MRI data correlate strongly with histology and enzymatic analysis. Magn Reson Imaging 10:773-778.

Aoyagi A, Saito H, Abe K, Nishiyama N (1998) Early impairment and late recovery of synaptic transmission in the rat dentate gyrus following transient forebrain ischemia in vivo. Brain Res 799:130-137.

Bederson JB, Pitts LH, Tsuji M, Nishimura MC, Davis RL, Bartkowski H (1986) Rat middle cerebral artery occlusion: evaluation of the model and development of a neurologic examination. Stroke 17:472-476.

Bolay H, Dalkara T (1998) Mechanisms of motor dysfunction after transient MCA occlusion: persistent transmission failure in cortical synapses is a major determinant. Stroke 29:1988-1994.

Calautti C, Leroy F, Guincestre JY, Baron JC (2001) Dynamics of motor network overactivation after striatocapsular stroke: a longitudinal PET study using a fixed-performance paradigm. Stroke 32:2534-2542.

Cramer SC, Bastings EP (2000) Mapping clinically relevant plasticity after stroke. Neuropharmacology 39:842-851.

Cuadrado ML, Egido JA, Gonzalez-Gutierrez JL, Varela-De-Seijas E (1999) Bihemispheric contribution to motor recovery after stroke: a longitudinal study with transcranial doppler ultrasonography. Cerebrovasc Dis 9:337-344.

Dijkhuizen RM, Ren J, Mandeville JB, Wu O, Ozdag FM, Moskowitz MA, Rosen BR, Finklestein SP (2001) Functional magnetic resonance imaging of reorganization in rat brain after stroke. Proc Natl Acad Sci USA 98:12766-12771.

Hallett M (2001) Plasticity of the human motor cortex and recovery from stroke. Brain Res Brain Res Rev 36:169-174.

Hamberg LM, Boccalini P, Stranjalis G, Hunter GJ, Huang Z, Halpern E, 
Weisskoff RM, Moskowitz MA, Rosen BR (1996) Continuous assessment of relative cerebral blood volume in transient ischemia using steady state susceptibility-contrast MRI. Magn Reson Med 35:168-173.

Herholz K, Heiss WD (2000) Functional imaging correlates of recovery after stroke in humans. J Cereb Blood Flow Metab 20:1619-1631.

Hoehn M, Nicolay K, Franke C, van der Sanden B (2001) Application of magnetic resonance to animal models of cerebral ischemia. J Magn Reson Imaging 14:491-509.

Inao S, Tadokoro M, Nishino M, Mizutani N, Terada K, Bundo M, Kuchiwaki H, Yoshida J (1998) Neural activation of the brain with hemodynamic insufficiency. J Cereb Blood Flow Metab 18:960-967.

Johansson BB (2000) Brain plasticity and stroke rehabilitation. The Willis lecture. Stroke 31:223-230.

Jones TA, Schallert T (1992) Overgrowth and pruning of dendrites in adult rats recovering from neocortical damage. Brain Res 581:156-160.

Jones TA, Kleim JA, Greenough WT (1996) Synaptogenesis and dendritic growth in the cortex opposite unilateral sensorimotor cortex damage in adult rats: a quantitative electron microscopic examination. Brain Res 733:142-148

Kawamata T, Dietrich WD, Schallert T, Gotts JE, Cocke RR, Benowitz LI, Finklestein SP (1997) Intracisternal basic fibroblast growth factor enhances functional recovery and up-regulates the expression of a molecular marker of neuronal sprouting following focal cerebral infarction. Proc Natl Acad Sci USA 94:8179-8184.

Lee RG, van Donkelaar P (1995) Mechanisms underlying functional recovery following stroke. Can J Neurol Sci 22:257-263.

Liepert J, Bauder H, Wolfgang HR, Miltner WH, Taub E, Weiller C (2000) Treatment-induced cortical reorganization after stroke in humans. Stroke 31:1210-1216.

Lin SP, Schmidt RE, McKinstry RC, Ackerman JJ, Neil JJ (2002) Investigation of mechanisms underlying transient T2 normalization in longitudinal studies of ischemic stroke. J Magn Reson Imaging 15:130-136.

Longa EZ, Weinstein PR, Carlson S, Cummins R (1989) Reversible middle cerebral artery occlusion without craniectomy in rats. Stroke 20:84-91.

Loubinoux I, Volk A, Borredon J, Guirimand S, Tiffon B, Seylaz J, Meric P (1997) Spreading of vasogenic edema and cytotoxic edema assessed by quantitative diffusion and T2 magnetic resonance imaging. Stroke 28:419-426.

Mandeville JB, Marota JJ, Kosofsky BE, Keltner JR, Weissleder R, Rosen BR, Weisskoff RM (1998) Dynamic functional imaging of relative cerebral blood volume during rat forepaw stimulation. Magn Reson Med 39:615-624.

Marshall RS, Perera GM, Lazar RM, Krakauer JW, Constantine RC, DeLaPaz RL (2000) Evolution of cortical activation during recovery from corticospinal tract infarction. Stroke 31:656-661.

Matsumoto K, Lo EH, Pierce AR, Wei H, Garrido L, Kowall NW (1995) Role of vasogenic edema and tissue cavitation in ischemic evolution on diffusion-weighted imaging: comparison with multiparameter MR and immunohistochemistry. AJNR Am J Neuroradiol 16:1107-1115.

Moseley ME, Cohen Y, Mintorovitch J, Chileuitt L, Shimizu H, Kucharczyk J, Wendland MF, Weinstein PR (1990) Early detection of regional cerebral ischemia in cats: comparison of diffusion- and T2-weighted MRI and spectroscopy. Magn Reson Med 14:330-346.

Nelles G, Spiekramann G, Jueptner M, Leonhardt G, Muller S, Gerhard H, Diener HC (1999) Evolution of functional reorganization in hemiplegic stroke: a serial positron emission tomographic activation study. Ann Neurol 46:901-909.

Netz J, Lammers T, Homberg V (1997) Reorganization of motor output in the non-affected hemisphere after stroke. Brain 120:1579-1586.
Nudo RJ, Friel KM (1999) Cortical plasticity after stroke: implications for rehabilitation. Rev Neurol 155:713-717.

Østergaard L, Weisskoff RM, Chesler DA, Gyldensted C, Rosen BR (1996) High resolution measurement of cerebral blood flow using intravascular tracer bolus passages. Part I: Mathematical approach and statistical analysis. Magn Reson Med 36:715-725.

Palmer GC, Peeling J, Corbett D, Del Bigio MR, Hudzik TJ (2001) T2weighted MRI correlates with long-term histopathology, neurology scores, and skilled motor behavior in a rat stroke model. Ann NY Acad Sci 939:283-296.

Paxinos G, Watson C (1997) The rat brain in stereotaxic coordinates. San Diego: Academic.

Pierpaoli C, Righini A, Linfante I, Tao-Cheng JH, Alger JR, Di Chiro G (1993) Histopathologic correlates of abnormal water diffusion in cerebral ischemia: diffusion-weighted MR imaging and light and electron microscopic study. Radiology 189:439-448.

Que M, Schiene K, Witte OW, Zilles K (1999a) Widespread up-regulation of $N$-methyl-D-aspartate receptors after focal photothrombotic lesion in rat brain. Neurosci Lett 273:77-80.

Que M, Witte OW, Neumann-Haefelin T, Schiene K, Schroeter M, Zilles K (1999b) Changes in GABA(A) and GABA(B) receptor binding following cortical photothrombosis: a quantitative receptor autoradiographic study. Neuroscience 93:1233-1240.

Reddy H, De Stefano N, Mortilla M, Federico A, Matthews PM (2002) Functional reorganization of motor cortex increases with greater axonal injury from CADASIL. Stroke 33:502-508.

Reese T, Porszasz R, Baumann D, Bochelen D, Boumezbeur F, McAllister KH, Sauter A, Bjelke B, Rudin M (2000) Cytoprotection does not preserve brain functionality in rats during the acute post-stroke phase despite evidence of non-infarction provided by MRI. NMR Biomed 13:361-370.

Rijntjes M, Weiller C (2002) Recovery of motor and language abilities after stroke: the contribution of functional imaging. Prog Neurobiol 66:109-122.

Rossini PM, Pauri F (2000) Neuromagnetic integrated methods tracking human brain mechanisms of sensorimotor areas "plastic" reorganisation. Brain Res Brain Res Rev 33:131-154.

Seil FJ (1997) Recovery and repair issues after stroke from the scientific perspective. Curr Opin Neurol 10:49-51.

Steinberg BA, Augustine JR (1997) Behavioral, anatomical, and physiological aspects of recovery of motor function following stroke. Brain Res Brain Res Rev 25:125-132.

Stroemer RP, Kent TA, Hulsebosch CE (1995) Neocortical neural sprouting, synaptogenesis, and behavioral recovery after neocortical infarction in rats. Stroke 26:2135-2144.

Tuor UI, Hudzik TJ, Malisza K, Sydserff S, Kozlowski P, Del Bigio MR (2001) Long-term deficits following cerebral hypoxia-ischemia in four-week-old rats: correspondence between behavioral, histological, and magnetic resonance imaging assessments. Exp Neurol 167:272-281.

van Gelderen P, de Vleeschouwer MH, DesPres D, Pekar J, van Zijl PC, Moonen CT (1994) Water diffusion and acute stroke. Magn Reson Med 31:154-163.

Verheul HB, Balazs R, Berkelbach van der Sprenkel JW, Tulleken CA, Nicolay K, Tamminga KS, van Lookeren Campagne M (1994) Comparison of diffusion-weighted MRI with changes in cell volume in a rat model of brain injury. NMR Biomed 7:96-100.

Weiller C (1998) Imaging recovery from stroke. Exp Brain Res 123:13-17.

Witte OW, Bidmon HJ, Schiene K, Redecker C, Hagemann G (2000) Functional differentiation of multiple perilesional zones after focal cerebral ischemia. J Cereb Blood Flow Metab 20:1149-1165. 